\title{
Osteopathische Behandlung bei akut traumatischen Zuständen: die Kraft der Flüssigkeiten
}

Marianne Mayer-Logeman

In den meisten Fällen kommen Patienten mit langjährigen oder chronischen Beschwerden in die osteopathische Praxis. Als Osteopathen versuchen wir, diesen auf den Grund zu gehen und die Bereiche, in denen sich die Gesundheit und Potency nicht mehr frei ausdrücken können, zu befreien. Ich freue mich immer, wenn sich ein Patient während oder nach einer akut traumatischen Situation meldet. Ähnlich wie bei der Behandlung von Babys kann man dann Einfluss auf ein zeitlich relativ kurz zurückliegendes Ereignis nehmen, wie es eine Geburt oder ein Trauma darstellen.

\section{Knochen als fluides System verstehen}

Ein langjähriger, 64-jähriger Patient rief mich aus dem Krankenhaus an, wo er nach einem Unfall stationär aufgenommen worden war. Er war als Radfahrer von einer Autofahrerin erfasst und über die Motorhaube geschleudert worden. Als Folge hatte er eine bimalleoläre Luxationsfraktur ( $\bullet$ Abb. 1) des linken oberen Sprunggelenks (OSG; Abb. 2), Kontusionsmarken am Innen- und Außenknöchel sowie einen Weichteilschaden Grad 2 nach Tscherne (tiefe kontaminierte Schürfung sowie Haut- oder Muskelkontusion durch direkte Krafteinwirkung, drohendes Kompartmentsyndrom mit mittelschweren bis schweren Frakturformen [z. B. Zweietagenfraktur der Tibia bei Stoßstangenanprall]) [1]. Zusätzlich und erst einige Tage später wurde eine stabile Radiusköpfchenfraktur diagnostiziert, die weder operativ noch im Sinne einer Immobilisierung versorgt werden musste.

Der Patient wollte gerne so bald wie möglich in die Praxis kommen, weil er schon früher nach akuten Eingriffen zur osteopathischen Behandlung bei mir war und gute Erfahrung damit gemacht hatte. Die Arbeit mit den Flüssigkeiten des ganzen Körpers oder lokal von bestimmten Geweben bietet sich in solchen Fällen hervorragend an. Dabei hat das Blutgefäßsystem eine herausragende Bedeutung, denn eine gute Wundheilung setzt eine gute Durchblutung voraus.

In der Osteopathie spielen die Flüssigkeiten eine zentrale Rolle. Wir bestehen aus Flüssigkeit in Flüssigkeit und W. G. Sutherland beschreibt es mit der Aussage: „Was ist

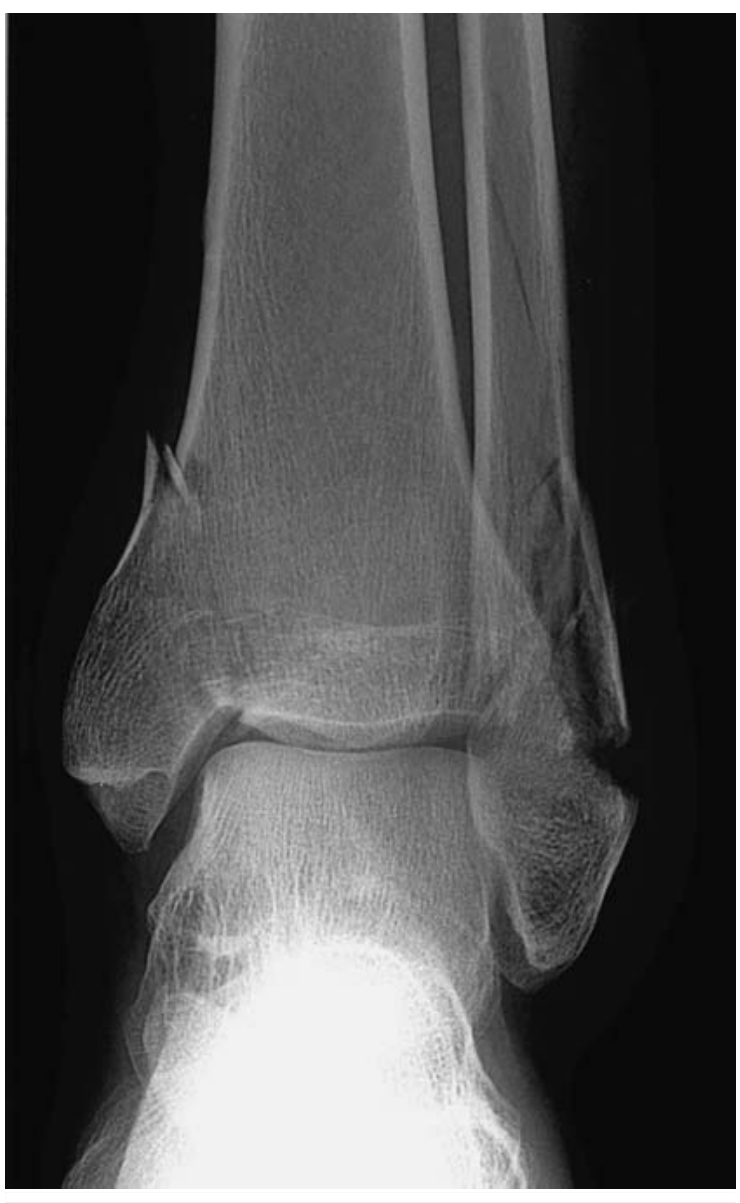

- Abb. 1 Bimalleoläre Sprunggelenksfraktur. (aus: [6]) 


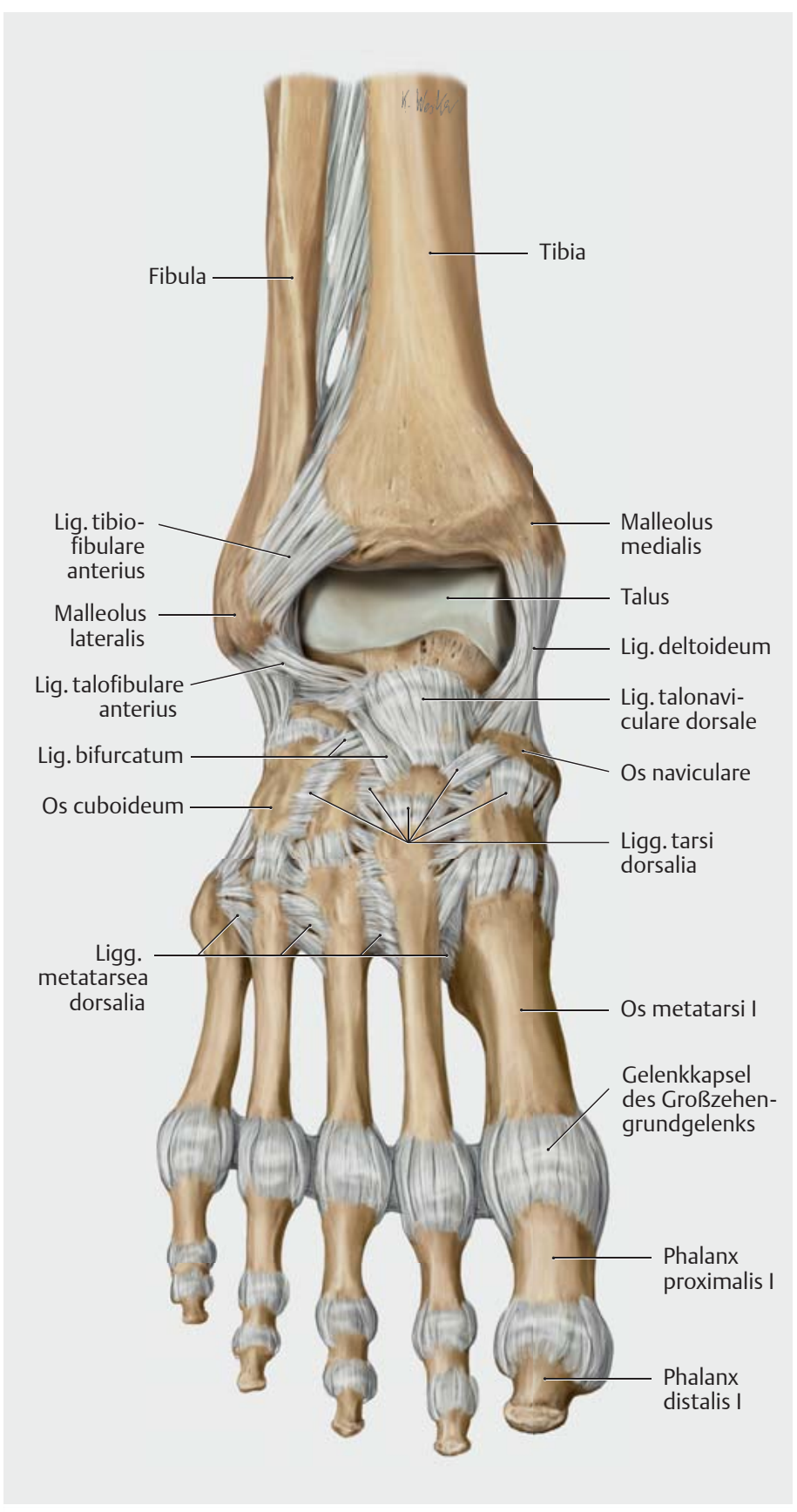

- Abb. 2 Bandapparat des oberen Sprunggelenks. (aus: [5])

Knochen, wenn nicht Flüssigkeit?" Von A. T. Still wird die Kraft der Flüssigkeiten den Stoffen und Substanzen zugeschrieben, die v.a. im Liquor cerebrospinalis enthalten sind. Sutherland meinte darüber hinaus, man sollte vielleicht effektiver das Gefühl für die „Säfte“ im ganzen Körper spüren lernen, um damit im Sinne der Gesundheit zu arbeiten [2].

Insbesondere bei akuten traumatischen Geschehen ist dieses Prinzip von enormer Bedeutung. Bei Verletzungen im Sinne von Gewebeunterbrechungen sind die Flüssig- keitsverbindungen gestört. Die Potenz der Reorganisation und Regeneration findet auf fluidischer Ebene statt und eine gute Vaskularisation ist dafür eine entscheidende Voraussetzung. Mit diesem Wissen im Hintergrund kann es sehr effektiv sein, wenn Patienten relativ früh zur Behandlung kommen, um diesen Heilungsprozess zu unterstützen und v.a. die Schock- und Traumamuster aufzulösen, damit diese der Heilung nicht im Wege stehen.

\section{Anamnese}

Der Patient hatte eine bimalleoläre OSG-Fraktur links, die operativ mit 2 Spongiosa-Halbgewindeschrauben am Innenknöchel, einer 8-Loch-1/3-Rohrplatte an der Fibula distal und einer Stellschraube versorgt worden war. Der Unterschenkel war mit elastischen Binden gewickelt und in einer dorsalen Unterschenkelhalbschale ruhiggestellt.

Der Patient war mit der operativen Versorgung des Beins zufrieden, sorgte sich aber um den rechten Ellbogen, da er als Musiker (Keybord und Gesang) um die Beweglichkeit des Arms fürchtete, und meinte, dass ich da am besten was „heilen“ könne.

2015 und 2016 war der Patient nach Umstellungsosteotomien bei Genu varum beidseits zur Behandlung bei mir gewesen. Die Knie waren von dem jetzigen Unfall nicht in Mitleidenschaft gezogen.

\section{Behandlung}

\section{Behandlung (8 Tage nach dem Unfall bzw. postoperativ)}

Der Patient machte einen sehr aufgeregten und nervösen Eindruck. Er wirkte von den Strapazen der Operation angestrengt. Das Gehen an Achselstützen, die er anstelle von Unterarmstützen zur Entlastung der Radiusköpfchenfraktur nutzte, ermüdete ihn zusätzlich.

Ich nahm vom Becken aus Kontakt zu seinem Körper auf und ging in Neutralität, um ohne Voreingenommenheit durch die Anamnese die Informationen seines Systems wahrzunehmen. Vor allem auf der Ebene des Fluid Body fühlte sich sein Körper noch im Schockzustand an und war sehr sympathikoton.

Obwohl für den Patienten der Ellenbogen im Vordergrund stand, wurde meine Aufmerksamkeit mehr auf das linke Bein gelenkt. Trotz des Verbands am linken Unterschenkel konnte ich spüren, dass sich der PRM in der Fibula und Tibia auf fluidischer Ebene nicht in longitudinaler Richtung ausdrücken konnte sowie auch intraossär und vaskulär kein harmonischer Ausdruck vorhanden war. Die Membrana interossea fühlte sich verzerrt an wie bei einem Strainmuster. 
In diesem Zustand war es nicht möglich, die Kraftvektoren des Traumas am Bein durch Positionieren in die entsprechende Kraftrichtung zu befreien. Über Directing the Tide (Lenkung der Tide) versuchte ich daher, das ganze Bein fluidisch besser an das Becken anzubinden - dies auch aus Sicht der Embryologie in Kontakt mit L5 [3].

Beim lokalen Kontakt mit dem Radius zeigte sich eine Kompression im proximalen Radioulnargelenk und auch hier war keine longitudinale Durchlässigkeit intraossär

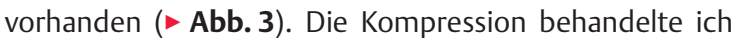
mittels BLT (Balanced Ligamentous Tension) und balancierte den gesamten Radius intraossär durch Directing the Tide in longitudinaler Richtung aus.

Von den Schultern bzw. Schulterblättern aus war das Schockmuster im gesamten Körper noch sehr deutlich erkennbar und die Mittellinie fühlte sich versetzt an. Aus völliger Neutralität heraus bot ich dem System Raum an. Im Sinne des Automatic Shifting spürte ich, dass die Fluida an verschiedenen Stellen arbeiteten, bis sich das Schockmuster spürbar veränderte und sich der PRM mit deutlich mehr Kraft ausdrücken konnte.

Nach der Behandlung erzählte der Patient, dass er das Unfallgeschehen noch einmal fühlen konnte, v.a. den Sturz über die Motorhaube. Er habe sich vorher wie eine verschrumpelte Pflaume gefühlt, irgendwie trocken, und während der Behandlung habe er „Plopps“ an verschiedenen Stellen des Körpers gespürt, bis er sich wieder gefüllt und ausgeglichener gefühlt habe. Insgesamt wirkte er nach der Behandlung ruhiger und weniger angestrengt.

Diese „Plopps“ werden im biodynamischen Sinne als „Zündung“ oder „Ignition“ beschrieben. Die Potency kann sich in den „trockenen“, nicht integrierten Bereichen des Körpers wieder ausdrücken [3].

\section{Behandlung (15 Tage postoperativ)}

Der Patient berichtete, dass er nach der 1. Behandlung ein viel freieres Gefühl im Ellenbogen habe, bei endgradigen Bewegungen spüre er noch etwas Druck im Gelenk proximal, kaum Schmerzen und leichte Einschränkungen distal. Sein Körpergefühl sei viel besser und er fühle sich widerstandsfähiger. Insgesamt wirkte er auch viel ruhiger und ausgeglichener.

Am Tag zuvor waren die Fäden am lateralen und medialen Knöchelbereich entfernt worden. Die Kontusionsbereiche waren reizfrei und verschorft. An der lateralen Naht hatte sich in der zurückliegenden Woche ein blasenartig entzündeter Bereich entwickelt. Zur Behandlung der Entzündung wurden 5 Tage Antibiotika verabreicht. Der Fuß war insgesamt deutlich abgeschwollen und weniger livide verfärbt. Eine Röntgenkontrolle des rechten Ellenbogens zeigte eine regelrechte Position des Radiusköpfchens.

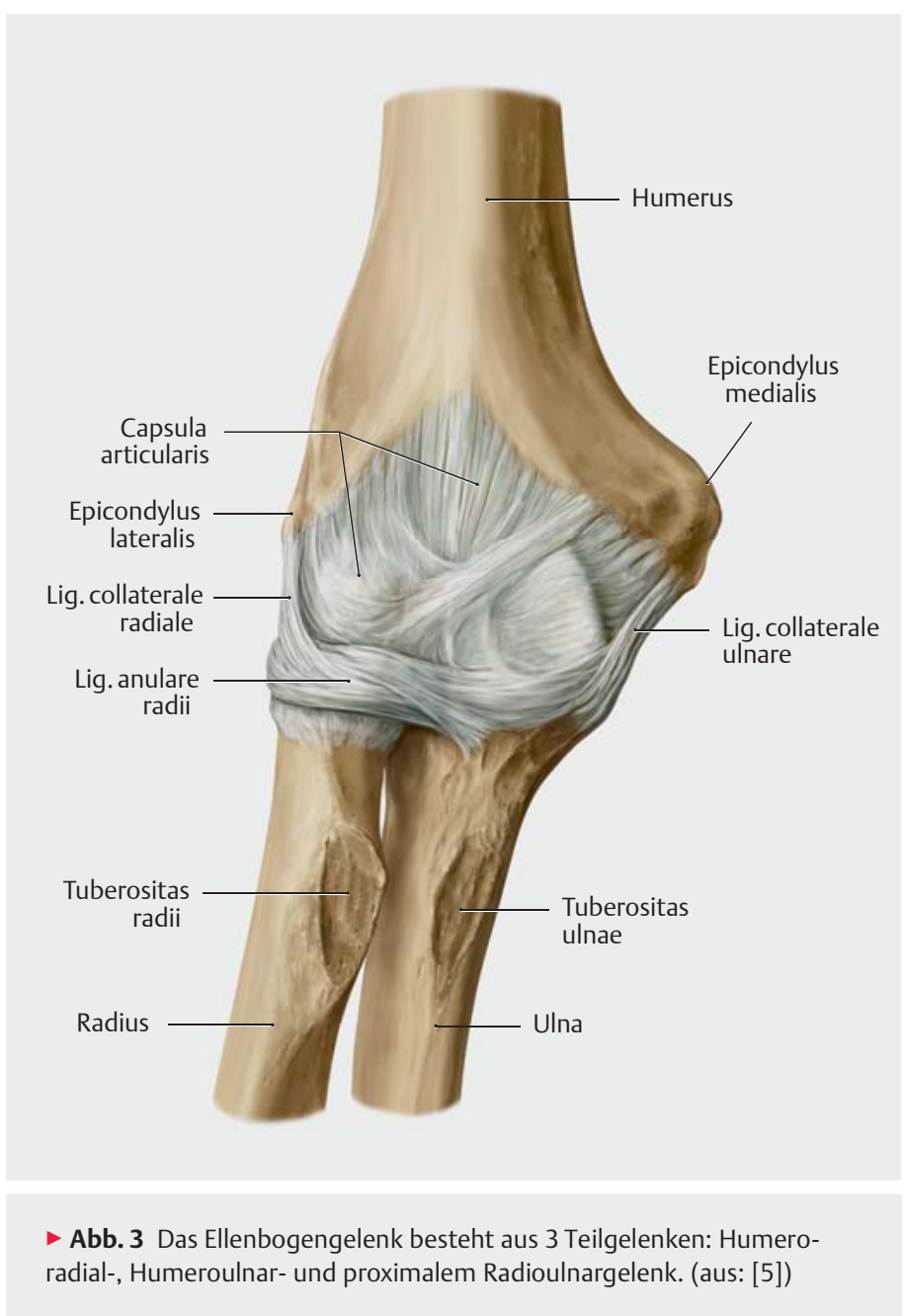

Ich ging wieder aus der Neutralität in Kontakt mit dem Körper des Patienten, dieses Mal von den Füßen aus. Der Ausdruck des PRM und der Fluida waren deutlich besser. Jetzt hatte ich den Eindruck, dass der Körper wieder atmen und Raum einnehmen konnte.

Der linke Fuß war noch sehr komprimiert, die Schraubenfixation tibiofibulär deutlich spürbar. Die fluidische Verbindung nach proximal fühlte sich besser an, ebenso die Midline der Tibia. Ich wurde zur tibiofibulären Verbindung und der Membrana interossea gelenkt und arbeitete mit lateraler Fluktuation in diesem Gebiet.

Der Ellenbogen zeigte keine Kompression mehr im proximalen Radioulnargelenk, aber einen weiterlaufenden Shift zur Apertura thoracica superior (ATS) von rechts nach links, was noch dem Aufprallmuster entsprach. Hier fragte das System nach einem Ausgleich der ATS indirekt/ fluidisch und auch die 3. Rippe zeigte sich im kostotransversalen Gelenk restriktiv, was ich mit BLT löste. Zur Verbesserung des lymphatischen Abflusses stimulierte ich alle kostotransversalen Verbindungen des Thorax mit GOT in Rückenlage. 
Zuvor hatte ich noch lokal mit dem Radiusköpfchen gearbeitet und die Osteoblasten sowie die zellulären Verbindung in diesem Gebiet visualisiert. Über das Anknüpfen an das embryologische Entwicklungsmuster verbesserte sich die Verbindung von der Hand bis zur Schulter des rechten Arms über C7 nach links.

\section{Behandlung (fast 4 Wochen postoperativ)}

Die Wundheilung im lateralen Narbenbereich war noch kritisch, denn direkt über dem Bereich, an dem die tibiofibuläre Fixationsschraube saß, trat immer noch Wundsekret aus. Die anfänglichen Nekrosestellen waren mit nur kleinen Schorfbereichen gut verheilt.

Der Ellenbogen fühlte sich viel freier an, der Patient hatte keine Knochenschmerzen mehr, fühlte jetzt muskuläre Spannungen bei Flexion und Supination. Er beschrieb diese Wahrnehmung als „Heilungsschmerz“.

Der Fuß war aktuell im Fußrücken-Zehen-Bereich geschwollen, weil der Patient ziemlich lange auf den Beinen war. Diese Überlastung zeigte sich als Kompression mit Stase im mittleren Tibiabereich und verringerte die fluidische Durchlässigkeit von Tibia und Fibula. Wieder arbeitete ich longitudinal mit einem Fluid Drive bis zum Ausgleich und balancierte die Membrana interossea.

Das Traumamuster im gesamten Fuß war wieder präsenter und die Metallfixationen als Bremse spürbar. Ich habe auf Ebene der Osteoblastenaktivierung versucht, eine vermehrte Kontinuität im Knochengewebe anzuregen. Bei allen Korrekturen beobachtete ich immer die zentrale Verbindung zu den großen Gefäßen des Beckens und habe diese ausgeglichen.

Nach der mehr ossären Arbeit wurde meine Aufmerksamkeit auf die schlecht heilende Narbenstelle gezogen. Hier habe ich mich auf zellulärer Ebene mit dem Gewebe verbunden und konnte eine kraftvolle fluidische Bewegung von tief innen nach außen spüren, ein Gefühl von Heilung und Verschluss. Diese Art zu arbeiten mag vielleicht manchem schwer vorstellbar erscheinen. Wir wissen aber, dass jedes Gewebe eine Ansammlung von Zellen mit anabolen und katabolen Stoffwechselprozessen innerhalb einzelner Zellen und untereinander ist. Und so wie man mit Zellverbänden z. B. im Sinne einer Extremität arbeiten kann, kann man auch die einzelnen Zellen visualisieren und in ihrem zyklischen Stoffwechsel ausgleichen [4].

\section{Behandlung (6 Wochen postoperativ)}

Der Patient berichtet, dass er nach der letzten Behandlung ein viel „durchlässigeres“ Gefühl in der gesamten unteren Extremität gehabt habe. Bei den Verbandswechseln sei eine deutliche Verbesserung des Wundbereichs festzustellen. Bis auf eine stecknadelkopfgroße Stelle zeige sich ein kompletter Verschluss der Narbe. Die Entfer- nung der Schraube, die nach 6 Wochen postoperativ geplant war, sei sicherheitshalber noch um eine Woche verschoben worden. Nach einer Überlastung am Wochenende, als er einen Auftritt hatte und viel gehen und stehen musste, habe das rechte Bein inzwischen zusätzlich geschmerzt. Die Achselstützen habe er gegen Unterarmgehstützen mit Zustimmung des Arztes ausgetauscht, da der Ellenbogen wieder voll belastbar sei. Diese Umstellung mache sich allerdings mit Schmerzen v.a. im distalen Radioulnargelenk bemerkbar.

Bei der Untersuchung am Ellenbogen war zu spüren, dass sich die geringgradige Mobilitätseinschränkung von proximal nach distal auswirkte. Grundsätzlich hatte der Radius aber eine gute Midline und keine Kompressionstendenz gegen das Olekranon mehr. Der primäre respiratorische Mechanismus (PRM) war mit gutem Ausdruck spürbar. Zur Balancierung arbeitete ich wieder mit der embryologischen Entwicklungsbewegung (Verbindung von C7 und beide Arme bis zum Mittelstrahl des Mittelfingers).

Beim Fuß konzentrierte ich mich wieder auf die zelluläre Ebene im Narbenbereich. Es war deutlich zu spüren, dass nur noch in der obersten Gewebeschicht Heilungsaktivitäten stattfanden, in der Tiefe herrschte Ruhe im Sinne einer guten vaskulären Versorgung, auch wenn die Unterbrechungen durch das Metall spürbar blieben. Ich balancierte das OSG mittels lateraler Fluktuation inkl. der Syndesmose und arbeitete longitudinal intraossär an Tibia und Fibula.

Die Behandlung schloss ich mit einem CV4 über das Okziput ab, weil der Patient vom Wochenende sehr erschöpft war. Damit konnte ich sein vitales Potenzial verbessern und der PRM drückte sich nach einem Stillpoint wieder besser aus.

\section{Behandlung (7 Wochen postoperativ)}

4 Tage vor der 5. Behandlung erfolgte die Entfernung der im distalen Drittel liegenden tibiofibulären Fixationsschraube. Die Narbe war noch mit Fäden versorgt, fast reizfrei und zeigte eine nur leichte Sekretabsonderung an der zuvor kritischen Stelle.

Der Vorfuß war insgesamt nicht mehr geschwollen, der mediale Knöchelbereich dafür aber deutlich. Der Patient deutete dies als Reaktion auf die begonnene Teilbelastung. Die Mobilität im OSG war nur noch endgradig eingeschränkt, was mit Blick auf das ursprüngliche Trauma zufriedenstellend war.

Spannend war für mich festzustellen, dass nun ohne die Fixationsschraube zwischen Tibia und Fibula das Traumamuster des Fußes in seinem ganzen Umfang deutlich zu spüren war. Das Muster war wie befreit von der mechanischen Barriere und ließ sich durch Arbeit mit den Kraft- 
vektoren des Traumas in Annäherung völlig auflösen. Jetzt fanden der gesamte Fuß und Unterschenkel eine noch bessere Midline, die sich auch bei der Fokussierung auf Knochenzellebene darstellte.

Zum Schluss balancierte ich die zu spürenden myofaszialen Verspannungen der Plantarsehne in Kontinuität zum Centrum tendineum des Beckens zum Ausgleich der Becken-Bein-Statik aus. Der PRM konnte sich nun über den ganzen Körper gut ausdrücken, das Gefühl von Gesundheit und Regeneration war wieder da.

Wir haben beschlossen, weitere Behandlungen in ca. 4wöchentlichen Abständen durchzuführen. Es sollten auch physiotherapeutische Behandlungen erfolgen.

\section{Fazit}

Die recht ausführliche Beschreibung der einzelnen Behandlungen und jeweiligen Befunde habe ich ganz bewusst gewählt. Als Dozentin mache ich immer wieder die Erfahrung, dass Studenten zu wenig genau hinschauen und einzelne Bereiche testen, um die Befunde mit dem zu verknüpfen, was der Körper bzw. das Gewebe des Patienten signalisiert.

Vielleicht denkt man im ersten Moment, dass man bei solch einem akuten Geschehen osteopathisch nichts machen kann. Aber ganz im Gegenteil kann ich aus Erfahrung sagen, dass man sehr detailliert Signale aus dem Gewebe bekommen kann, die man zu hören lernen und entsprechend darauf reagieren sollte. Das osteopathische Prinzip, nach der Gesundheit zu schauen und sich nicht von der Krankheit lenken zu lassen, hilft in solchen Fällen enorm. Denn in einem noch so verletzten Körper steckt immer noch genug Potency, die zu kompensieren und auch zu heilen hilft. Dabei kann es eine wunderbare Herausforderung sein, sich trotz der Fokussierung auf das Detail davon nicht gefangennehmen zu lassen, sondern immer das Gesamte im Blick zu haben. Dieses Ganze im Sinne der embryologischen Entwicklung zu sehen, kann dabei ein guter Leitfaden sein, was James Jealous sehr schön ausdrückt: „Die Kräfte der embryologischen Entwicklung werden beim Erwachsenen zu den Kräften der Heilung. “ [3]
Und letztendlich können wir uns und den Patienten entlasten, indem wir dem Faktor Zeit ausreichend Wert beimessen. Auch wenn man in akuten Fällen eher „akut“, also engmaschiger, behandelt, spürt man, dass das Gewebe die Zeit für Regenerations- und Reparaturprozesse benötigt. Die Patienten sind sehr dankbar dafür zu hören, dass sie eine Pause zwischen allen Therapien machen dürfen und auch sollten, in dem Vertrauen, dass sich die Gesundheit des Organismus in dieser Pause noch besser ausdrücken kann.

\section{Autorinnen/Autoren}

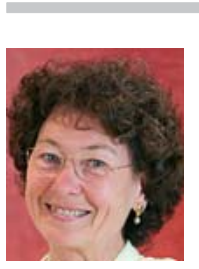

\section{Marianne Mayer-Logeman}

graduierte 1996 am College Sutherland Hamburg. Neben der Praxistätigkeit unterrichtet sie regelmäßig am CS und der ECOM Hamburg das Fach kraniale Osteopathie. Sie ist Mitglied der Fakultät des Sutherland Cranial College of Osteopathy.

\section{Korrespondenzadresse}

Marianne Mayer-Logeman D.O. M.R.O. FSCCO HP

Saarstr. 13

26121 Oldenburg

mayer.marianne@t-online.de

Literatur

[1] www.traumascores.com/index.php/kl2-2/40-allgemein4/ $116-115$

[2] Sutherland WG. Das große Sutherland-Kompendium. Pähl: Jolandos; 2004

[3] Shaver T. Eigene Mitschriften aus dem Kurs „Biodynamic“; 2004-2012

[4] Marris T. Eigene Mitschriften aus dem Workshop in Bad Nauheim; 2016

[5] Schünke M, Schulte E, Schumacher U. Prometheus. LernAtlas der Anatomie. Allgemeine Anatomie und Bewegungssystem. Illustrationen von M. Voll und K. Wesker. 4. Aufl. Stuttgart: Thieme; 2018

[6] Stäbler A, Ertl-Wagner B, Hrsg. Radiologie-Trainer: Bewegungsapparat. 3. Aufl. Stuttgart: Thieme; 2015

Bibliografie

DOI https://doi.org/10.1055/a-0732-3763

DO - Deutsche Zeitschrift für Osteopathie 2019; 17: 15-19

(c) Georg Thieme Verlag KG Stuttgart · New York ISSN 1610-5044 